\title{
Role of miRNAs in the response to anticancer
}

\section{therapy}

\section{"...studies have linked a number of miRNAs to resistance or response to anticancer therapies in various tumor types."}

\section{KEYWORDS: cancer $\approx$ drug resistance $\approx$ miRNA $\approx$ pharmaco-miRNomics}

miRNA (miR) are endogenous, small, singlestranded, ncRNAs that play important roles in multiple cellular processes [1], and are evolutionarily conserved in sequence between distantly related organisms, suggesting they are essential biomolecules. miRs act as negative regulators of gene expression at the post-transcriptional level, through binding to partially complementary sequence motifs present mainly in the 3'-UTRs of corresponding mRNAs, thereby causing either translational repression and/or target mRNA degradation. Recently it has been reported that conserved miR binding sites also exist in the open reading frame and 5'-UTR regions of target mRNAs [2]. There is a 'many-to-many relationship' between miRs and mRNAs with potential phenotypic consequences, as a single miR can simultaneously deregulate multiple mRNAs, while a single mRNA can be targeted by several miRs [3]. Since their discovery in 1993, there has now been a 'miR revolution' in molecular oncology and most human cancers have been profiled and classified by distinctive miR signatures. Alterations in miR expression and function contribute to cancer development through a multitude of mechanisms and miRs are able to act as tumor suppressors (TSG-miRs) or oncogenes (onco-miRs) in a tissue/tumor specific manner [4]. From this, the diagnostic value and therapeutic potential of miRs has been realized.

Chemotherapy is widely used in the treatment of various types of cancer and adequate response is crucial for prolonging patient survival and improving quality of life. Despite the development of novel anticancer agents, drug resistance frequently hampers successful treatment and many tumors are still refractory to chemotherapy. Chemoresistance can be classified as either intrinsic or acquired. The intrinsic type of resistance refers to factors already present (including a combination of genetic and biochemical factors such as the genetic variability of the cancer cell population and the poor ability to transport the drug into the cells) that would make the intended anticancer therapy futile. Acquired resistance however develops over time, after exposure to chemotherapy, when tumors that were initially sensitive to a particular drug quickly develop resistance, often due to overgrowth of drug-resistant tumor cells [5]. The acquisition of chemoresistance correlates with increased aggressiveness and poor prognosis. Recognized causes of resistance to anticancer therapy include genetic (random drug-induced mutational events), epigenetic (drug-induced non-mutational alterations affecting the expression and function of critical genes) and karyotypic changes (drug-induced chromosomal abnormalities) [5]. Resistance is considered to be a multifactorial phenomenon and mechanisms include problems with drug delivery, drug metabolism and molecular changes in tumors affecting drug sensitivity [6]. Understanding the mechanisms involved is crucial to developing more effective treatments and better utilizing currently available agents.

The identification of biomarkers that can accurately classify tumors and help to improve the initial choice of anticancer treatment, by avoiding intrinsic resistance and forecasting the development of acquired resistance is paramount. Experimental evidence demonstrates that miRs are involved in the chemoresistant phenotype of tumor cells and miR-expression profiling can be correlated with the evolution of anticancer drug resistance. This miR-mediated form of drug resistance adds yet another cause of anticancer treatment failure [6] and suggests a role for tumor miRs, not only as diagnostic and predictive biomarkers, but also as targets that must be exploited to either optimize treatment response and/or produce direct antitumor

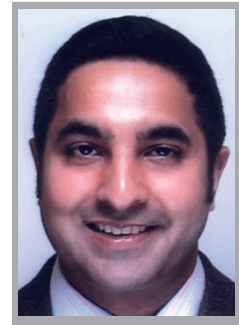

Adam E Frampton

Author for correspondence: HPB Surgical Unit, Department of Surgery \& Cancer, Imperial College, Hammersmith Hospital Campus, Du Cane Road, London, W12 OHS, UK a.frampton@imperial.ac.uk

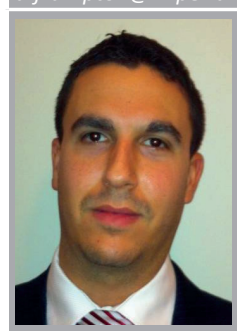

Jonathan Krell

Division of Oncology, Department of Surgery \& Cancer, Imperial Centre for Translational \& Experimental Medicine (ICTEM), Imperial College, Hammersmith Hospital Campus, Du Cane Road, London, W12 ONN, UK Elisa Giovannetti Department of Medical Oncology, VU University Medical Center, De Boelelaan 1117, 1081 HV Amsterdam, The Netherlands

\section{Long R Jiao}

HPB Surgical Unit, Department of Surgery \& Cancer, Imperial College, Hammersmith Hospital Campus, Du Cane Road, London, W12 OHS, UK

Justin Stebbing Department of Oncology, Imperial College Healthcare NHS Trust, Charing Cross Hospital, 1st Floor, E Wing, Fulham Palace Road, London, W6 8RF, UK

Future $\because$ Medicine part of 
effects. Several studies have shown the correction of specific deregulated miRs, using miR mimics or inhibitors, can normalize the gene regulatory network and signaling pathways, and potentially reverse unwanted phenotypes in cancerous cells [7]. miR-based therapeutics therefore provide a very attractive antitumor approach for integrated cancer therapy. These strategies may allow us to overcome chemoresistance via a synergistic interaction with anticancer drugs, by modulation of key proteins involved in drug activity, thereby improving the clinical outcome of cancer patients [8].

Tumors presenting clinically as the same type or subtype can be very different at the molecular and miR level and therefore behave differently in response to anticancer therapy. Interestingly, the expression of various miRs is also affected by some anticancer agents, therefore providing an intertwined scenario whereby miRs can be modulated by drugs which in turn affects the chemosensitivity of cancer cells [9]. Early tumorprofiling studies have led to an understanding of differential miR expression between clinical cases and molecular subtypes, the correlation with response to anticancer cytotoxic therapies, radiotherapy and radical surgery will allow for more robust prognostic signatures and further individualization of care. Here we consider some of the recent discoveries in the role of miRs in anticancer drug resistance.

The NCI-60, a panel of 60 diverse human cancer cell lines, has been used by the NCI to screen $>100,000$ chemical compounds for anticancer activity. These well studied cancer cell lines have been subjected to every 'omic analysis and have recently been extensively profiled for their miRNome (the entire miRNA content of cells, tissue or tumor) and transcriptome in response to several anticancer cytotoxics $[10,11]$. Significant correlations have been demonstrated between miR-expression profiles and drug potency patterns, suggesting that miRs may provide a critical link for understanding mechanisms involved in chemosensitivity and chemoresistance [10].

Other studies have linked a number of miRs to resistance or response to anticancer therapies in various tumor types. Pancreatic cancer is one of the deadliest cancers and over the past 15 years there has been little tangible progress in terms of treatments. The pathological role of miRs is not well understood, but miR-21 is thought to be significant $[12,13]$. The inhibition of miR-21 increased sensitivity of pancreatic ductal adenocarcinoma (PDAC) cells to gemcitabine [12] and two clinical studies provide further evidence that miR-21 affects chemosensitivity in pancreatic cancer. The first study using 81 PDAC samples from patients treated with gemcitabine, found high miR-21 expression was associated with poorer overall survival in both the adjuvant and metastatic settings [14], and a subsequent study demonstrated a correlation between low miR-21 expression and improved outcome in patients with localized PDAC treated with adjuvant gemcitabine or 5-fluorouracil chemotherapy [15].

Multidrug resistance (MDR) in specific MCF-7 breast cancer cell lines was associated with reduced levels of miR-326 [16] and miR451 [17] leading to upregulated expression of the MRP-1/ABCC1 and the MDR1, respectively, and reconstitution of miR-451 expression sensitized cells to doxorubicin. Furthermore, the miR-17-92 and miR-106a-363 families control estrogen receptor- $\alpha$ transcription and cellular responses to estrogen in breast cancer and their expression levels may predict response to antiestrogens [18]. A recent retrospective study of over 200 patients with metastatic breast cancer treated with tamoxifen in the first-line setting found that high tumor miR-26a levels (but not miR-101 levels) were associated with improved clinical benefit and time to progression [19].

In hepatocellular carcinoma, the let-7 family targeted $\mathrm{Bcl}-\mathrm{X}_{\mathrm{L}}$ in cell lines and overexpression of these miRs increased sorafenib-induced apoptosis in cell culture experiments [20]. Furthermore, Ji et al. demonstrated an association between improved response and low miR-26 expression in over 200 hepatocellular carcinoma patients treated with IFN- $\alpha$ [21]. Studies in human ovarian cancer cell lines demonstrated an association between taxol resistance and increased expression of MDR1/P-gylcoprotein due to downregulation of miR-27a [22]. In a study of 37 stage III ovarian cancer patients, seven miRs, including miR$27 \mathrm{a}$, were significantly differentially expressed in tumors from platinum-resistant versus -sensitive patients. High miR-27a expression was associated with a particularly poor prognosis in terms of survival [23]. Furthermore, miR-200b (belonging to the family of miRs involved in epithelialto-mesenchymal transition) reversed chemoresistance of docetaxel-resistant human lung adenocarcinoma cells [24], and both miR-415 and miR-20a have been implicated in chemotherapeutic resistance in colorectal cancer cell lines $[25,26]$.

In a study of fludarabine-treated chronic lymphocytic leukemia patients, $37 \mathrm{miRs}$ accurately distinguished responders from nonresponders, 
with miR-21, miR-148a and miR-122 being more highly expressed in nonresponding patients [27]. In a similar study involving 50 chronic lymphocytic leukemia patients, fludarabine resistance was associated with decreased miR-29a and increased miR-181a expression [28].

The study of pharmacogenomics has become essential for successful drug discovery and offers widespread public health benefits [29]. For anticancer drugs, it provides an opportunity to develop superior, more targeted therapies with reduced toxicities. We believe 'pharmacomiRNomics', a combination of pharmacology and miRNomics [30], referring to the future analysis of tumor miRNomes relevant to drug target discovery, metabolism, efficacy and toxicity, will play an even more important role in the multidisciplinary effort to treat cancer. This approach should lead to better tumor classification, prognostic stratification and ultimately individualized treatment strategies.

However, from previous studies on the role of miRNAs in resistance to conventional chemotherapies, it is evident that similar to gene profiling studies, most emerging miRNA signatures are not fully overlapping. These results might be explained by different tumor specimens (e.g., frozen vs paraffin-embedded, micro- vs macro-dissected); experimental platforms (e.g., quantitative PCR vs miRNA arrays or in situ hybridization); tumor histology (type and stage) and treatment regimens, as well as by small sample size and lack of multivariate analysis or correction for multiple testing. In this regard, the achievement of a general consensus on the techniques and controls to be used and the conduction of studies on a larger scale, with associated pathobiological and clinical information, are warranted for the proper validation of specific miRNAs as new biomarkers of drug activity.

New technologies now offer more powerful methods to study interactions between miRs and genes, including the possibility of characterizing multiplicity, redundancy and the additive effect of miRs in these complex networks [31]. These include next-generation sequencing, combined with other methods, such as high-throughput sequencing of RNA isolated by crosslinking immunoprecipitation (HITS-CLiP, also known as Clip-Seq) [32], which can be used to decode miR-mRNA and protein-RNA interaction maps; and photoactivatable-ribonucleosideenhanced crosslinking and immunoprecipitation (PAR-CliP), which can identify, on a transcriptome-wide scale, binding sites of RNA-binding proteins, their binding motifs and preferences [33].
Together, these will provide a unique opportunity for molecular biologists to fully understand the miRNome structure and function in human cancers and translate their findings into improving anticancer drug response.

Currently, substantial preclinical and clinical evidence exists in several tumor types illustrating how miRs are able to predict and/or abrogate the chemoresistance of individual malignancies to different anticancer therapies and can therefore potentially help guide oncologists. Although these data are promising, larger prospective trials are required to validate the role of miRs in this capacity, but such studies should lead to significant changes in clinical treatment algorithms. Additionally, much data are derived from work using cancer cell lines, which have been removed from their in vivo environment, and therefore cannot be considered accurate surrogates for clinical tumors. Therefore, further animal studies assessing the mechanisms of these miRs in vivo are urgently required. Although the development of effective miR-modulating therapies has been challenging and important issues mainly relating to the development of new approaches for the in vivo delivery of miRNAmodulating molecules need to be addressed, recent advances using tiny locked nucleic acids [34] and lipid-based nanoparticles [35] are soon to make miR-based therapeutics possible.

\section{"...future analysis of tumor miRNomes relevant to drug target discovery, metabolism, efficacy and toxicity, will play an even more important role in the multidisciplinary effort to treat cancer."}

The increasing availability and reduced cost of RNA profiling will enable oncologists to stratify patients based on specific miR biomarkers before anticancer treatment, allowing the optimization of clinical outcomes through effective personalization of treatment. The implementation of personalized cancer care by incorporating tumor miRNomic data is now becoming a reality.

Financial \& competing interests disclosure

The authors have no relevant affliations or financial involvement with any organization or entity with a financial interest in or financial conflict with the subject matter or materials discussed in the manuscript. This includes employment, consultancies, honoraria, stock ownership or options, expert testimony, grants or patents received or pending, or royalties.

No writing assistance was utilized in the production of this manuscript. 


\section{References}

1 Croce CM. Causes and consequences of microRNA dysregulation in cancer. Nat. Rev. Genet. 10(10), 704-714 (2009).

2 Forman JJ, Legesse-Miller A, Coller HA. A search for conserved sequences in coding regions reveals that the let-7 microRNA targets Dicer within its coding sequence. Proc. Natl Acad. Sci. 105(39), 14879-14884 (2008).

3 Jayaswal V, Lutherborrow M, Ma DD, Yang $\mathrm{YH}$. Identification of microRNA-mRNA modules using microarray data. $B M C$ Genomics 12, 138 (2011).

4 Kasinski AL, Slack FJ. MicroRNAs en route to the clinic: progress in validating and targeting microRNAs for cancer therapy. Nat. Rev. Cancer 11(12), 849-864 (2011).

5 Allen KE, Weiss GJ. Resistance may not be futile: microRNA biomarkers for chemoresistance and potential therapeutics. Mol. Cancer Ther. 9(12), 3126-3136 (2010).

6 Zheng T, Wang J, Chen X, Liu L. Role of microRNA in anticancer drug resistance. Int. J. Cancer 126(1), 2-10 (2010).

7 Wang V, Wu W. MicroRNA-based therapeutics for cancer. BioDrugs 23(1), 15-23 (2009).

8 Giovannetti E, Erozenci A, Smit J, Danesi R, Peters GJ. Molecular mechanisms underlying the role of microRNAs (miRNAs) in anticancer drug resistance and implications for clinical practice. Crit. Rev. Oncol. Hematol. 81(2), 103-122 (2012).

9 Fanini F, Vannini I, Fabbri M. MicroRNAs and drug modulation in cancer: an intertwined new story. Front. Biol. 6(5), 351-356 (2011).

10 Blower PE, Verducci JS, Lin S et al. MicroRNA expression profiles for the NCI-60 cancer cell panel. Mol. Cancer Ther. 6(5), 1483-1491 (2007).

11 Reinhold WC, Sunshine M, Liu H et al. CellMiner: a web-based suite of genomic and pharmacologic tools to explore transcript and drug patterns in the NCI-60 cell line set. Cancer Res. 72(14), 3499-3511 (2012).

12 Park JK, Lee EJ, Esau C, Schmittgen TD. Antisense inhibition of microRNA-21 or -221 arrests cell cycle, induces apoptosis, and sensitizes the effects of gemcitabine in pancreatic adenocarcinoma. Pancreas 38(7), E190-E199 (2009).
13 Jiao LR, Frampton AE, Jacob J et al. MicroRNAs targeting oncogenes are down-regulated in pancreatic malignant transformation from benign tumors. PLoS One 7(2), e32068 (2012).

14 Giovannetti E, Funel N, Peters GJ et al. MicroRNA-21 in pancreatic cancer: correlation with clinical outcome and pharmacologic aspects underlying its role in the modulation of gemcitabine activity. Cancer Res. 70(11), 4528-4538 (2010).

15 Hwang JH, Voortman J, Giovannetti E et al. Identification of microRNA-21 as a biomarker for chemoresistance and clinical outcome following adjuvant therapy in resectable pancreatic cancer. PLoS One 5(5), e10630 (2010).

16 Liang Z, Wu H, Xia J et al. Involvement of miR-326 in chemotherapy resistance of breast cancer through modulating expression of multidrug resistance-associated protein 1. Biochem. Pharmacol. 79(6), 817-824 (2010).

17 Kovalchuk O, Filkowski J, Meservy J et al. Involvement of microRNA-451 in resistance of the MCF-7 breast cancer cells to chemotherapeutic drug doxorubicin. Mol. Cancer Ther. 7(7), 2152-2159 (2008).

18 Castellano L, Giamas G, Jacob J et al. The estrogen receptor- $\alpha$-induced microRNA signature regulates itself and its transcriptional response. Proc. Natl Acad. Sci. USA 106(37), 15732-15737 (2009).

19 Jansen MP, Reijm EA, Sieuwerts AM et al. High miR-26a and low CDC2 levels associate with decreased EZH2 expression and with favorable outcome on tamoxifen in metastatic breast cancer. Breast Cancer Res. Treat. 133(3), 937-947 (2011).

20 Shimizu S, Takehara T, Hikita $\mathrm{H}$ et al. The let-7 family of microRNAs inhibits Bcl-xL expression and potentiates sorafenib-induced apoptosis in human hepatocellular carcinoma. J. Hepatol. 52(5), 698-704 (2010).

21 Ji J, Shi J, Budhu A et al. MicroRNA expression, survival, and response to interferon in liver cancer. N. Engl. J. Med. 361(15), 1437-1447 (2009).

22 Li Z, Hu S, Wang J et al. MiR-27a modulates MDR1/P-glycoprotein expression by targeting HIPK2 in human ovarian cancer cells. Gynecol. Oncol. 119(1), 125-130 (2010).

23 Eitan R, Kushnir M, Lithwick-Yanai G et al. Tumor microRNA expression patterns associated with resistance to platinum based chemotherapy and survival in ovarian cancer patients. Gynecol. Oncol. 114(2), 253-259 (2009).

24 Feng B, Wang R, Song HZ, Chen LB. MicroRNA-200b reverses chemoresistance of docetaxel-resistant human lung adenocarcinoma cells by targeting E2F3. Cancer 118(13), 3365-3376 (2011) (Epub ahead of print).

25 Chai H, Liu M, Tian R, Li X, Tang H. miR-20a targets BNIP2 and contributes chemotherapeutic resistance in colorectal adenocarcinoma SW480 and SW620 cell lines. Acta Biochem. Biophys. Sinica 43(3), 217-225 (2011).

26 Bitarte N, Bandres E, Boni V et al. MicroRNA-451 is involved in the self-renewal, tumorigenicity, and chemoresistance of colorectal cancer stem cells. Stem Cells 29(11), 1661-1671 (2011).

27 Ferracin M, Zagatti B, Rizzotto L et al. MicroRNAs involvement in fludarabine refractory chronic lymphocytic leukemia. Mol. Cancer 9, 123 (2010).

28 Moussay E, Palissot V, Vallar L et al. Determination of genes and microRNAs involved in the resistance to fludarabine in vivo in chronic lymphocytic leukemia. Mol. Cancer 9, 115 (2010).

29 Hudson J. Pharmacogenomics: where does Britain stand? Pharmacogenomics 13(1), 1-3 (2012).

30 Ghosh Z, Chakrabarti J, Mallick B. miRNomics - the bioinformatics of microRNA genes. Biochem. Biophys. Res. Commun. 363(1), 6-11 (2007).

31 Malumbres M. miRNAs versus oncogenes: the power of social networking. Mol. Syst. Biol. 8,569 (2012).

32 Chi SW, Zang JB, Mele A, Darnell RB. Argonaute HITS-CLIP decodes microRNAmRNA interaction maps. Nature 460(7254), 479-486 (2009).

33 Hafner M, Landthaler M, Burger L et al. PAR-CliP - a method to identify transcriptome-wide the binding sites of RNA binding proteins. J. Vis. Exp. 41, pii: 2034 (2010).

34 Obad S, dos Santos CO, Petri A et al. Silencing of microRNA families by seed-targeting tiny LNAs. Nat. Genet. 43(4), 371-378 (2011).

35 Huang L, Liu Y. In vivo delivery of RNAi with lipid-based nanoparticles. Ann. Rev. Biomed. Eng. 13(1), 507-530 (2011). 